\title{
Encadrer la médiation : le cas de la prononciation
}

\section{Anne Péchou et Anthony Stenton}

\section{OpenEdition}

\section{Journals}

Édition électronique

URL : http://journals.openedition.org/asp/1919

DOI : 10.4000/asp.1919

ISBN : 978-2-8218-0384-8

ISSN : 2108-6354

Éditeur

Groupe d'étude et de recherche en anglais de spécialité

Édition imprimée

Date de publication : 1 octobre 2001

Pagination : 153-162

ISSN : 1246-8185

Référence électronique

Anne Péchou et Anthony Stenton, «Encadrer la médiation : le cas de la prononciation », ASp [En ligne], 31-33 | 2001, mis en ligne le 13 novembre 2010, consulté le 20 avril 2019. URL : http:// journals.openedition.org/asp/1919; DOI : 10.4000/asp.1919

Ce document a été généré automatiquement le 20 avril 2019

Tous droits réservés 


\title{
Encadrer la médiation : le cas de la prononciation
}

\author{
Anne Péchou et Anthony Stenton
}

1 Au cours des vingt dernières années, l'enseignement des langues vivantes, sous l'effet des méthodes communicatives, s'est davantage centré sur l'expression orale. Or, si des méthodes d'enseignement mieux adaptées (Péchou 2001) ont eu pour conséquence une amélioration notable de la fluidité, la qualité de la prononciation laisse beaucoup à désirer : phonétique approximative, accents toniques déplacés et mélodie désespérément plate, dont les effets combinés gênent considérablement l'auditeur quand ils ne rendent pas le message incompréhensible. Plaidant la cause d'un enseignement intégré de la phonétique dans un cours à orientation communicative destiné aux formateurs, CelceMurcia fait référence à un niveau seuil de prononciation qui devrait constituer l'objectif à atteindre par des locuteurs non-natifs pour être compris :

If they fall below this threshold level, they will have oral communication problems no matter how excellent and extensive their control of English grammar and vocabulary might be. (1996:7)

2 Elle souligne qu'un des objectifs de l'apprentissage devrait être de dépasser ce seuil afin que les erreurs de prononciation n'entravent pas la communication. Pourtant, phonétique et intonation font figure de parent pauvre dans nos cours, en partie parce que les enseignants sont peu formés dans une matière réputée ardue et rébarbative, et qu'ils ont d'autres priorités que les corrections phonétiques et phonologiques. Germain et Martin (2000) invoquent aussi le manque de dialogue entre chercheurs et enseignants insuffisamment formés, et des outils de remédiation peu attrayants et guère innovants. Si certaines méthodes sur cédérom offrent de courtes activités de sensibilisation (ex. Pronunciation Power ${ }^{1}$ ), il n'y a à ce jour ni traitement complet, ni système-auteur sur le marché. L'offre sur Internet s'améliore de façon quotidienne mais la lenteur des téléchargements, la perte de qualité dans les fichiers-son comprimés avec MP3, Real Audio ou WMA, et l'instabilité des programmes, constituent toujours de vrais obstacles. Soulignons en outre la pénurie sur le marché de logiciels qui permettent la classification des sons en voyelles et consonnes. 
Dans la logique d'un travail de recherche visant à améliorer l'expression orale d'apprenants de niveau intermédiaire à avancé, nous avons été conduits à réfléchir sur l'usage des technologies pour effectuer une sensibilisation à la phonétique et à l'intonation. Que peut-on exactement attendre des NT pour faciliter cet apprentissage délicat? Ce questionnement revêt toute son importance dans la perspective des cyberlicences $^{2}$ qui se mettent en place. Dans cette optique, il ne suffit plus simplement de remédiation comme supplément à un cours en présentiel. Il nous faut un outil d'autoformation adapté à un cours en ligne. Nous envisageons qu'il puisse également servir à l'auto-diagnostic.

4 Laissant pour le moment de côté la discussion du travail en cours sur la représentation à l'écran des schémas intonatifs qui sera l'étape suivante, nous traiterons ici seulement de l'élaboration d'exercices destinés à corriger deux types d'erreurs: discrimination voyelles/diphtongues, et place de l'accent tonique dans le mot.

5 Notre corpus est constitué de relevés de fautes de prononciation produites par les étudiants de l'Université des Sciences Sociales au cours de jeux de rôle et d'exposés, complétés par des glossaires des termes les plus fréquents en droit et en économie, compilés par les enseignants des divers domaines de spécialité dans le cadre du projet TUSSELE (voir Péchou \& Stenton 1999b et Stenton 2000).

6 Grâce aux activités proposées dans le laboratoire multimédia de l'établissement, ou sur intranet, nous espérons limiter les erreurs de prononciation des mots les plus fréquents et aider l'étudiant à dégager des règles.

\section{Voyelles et diphtongues}

7 Nous n'avons pas, dans un premier temps, cherché à élaborer une méthode complète de correction phonétique. Nous nous sommes attachés au traitement des fautes occasionnées par la similitude entre les deux langues, telles la confusion entre [I] et [aI], et les fautes d'utilisation des autres diphtongues [

eI], [aU] [U] dont la présence n'est souvent pas perçue, ou qui sont remplacées par un son simple, ou un son français.

Le matériel développé aborde la remédiation par quelques exercices de classification au cours desquels l'étudiant peut vérifier ses hypothèses par tâtonnements en maniant la souris. Nous parlerons ensuite du problème de la création par l'enseignant.

\subsection{Choix binaire entre les sons [al] et [l]}

10 Dans la colonne de gauche est présentée une liste de mots offrant des difficultés aux francophones. La voyelle mal prononcée a été soulignée. Selon que l'on clique avec le bouton droit ou gauche de la souris, le mot se place dans l'une des deux colonnes sous le symbole phonétique. Si le mot est bien placé, il s'affiche en caractères gras et on l'entend prononcé, ce qui fournit un renforcement positif. Mal placé, il apparait en italique et il n'y a pas de son (Fig.1). 
Fig. 1. Coping with vowel sounds, le cas de [al] et [1].

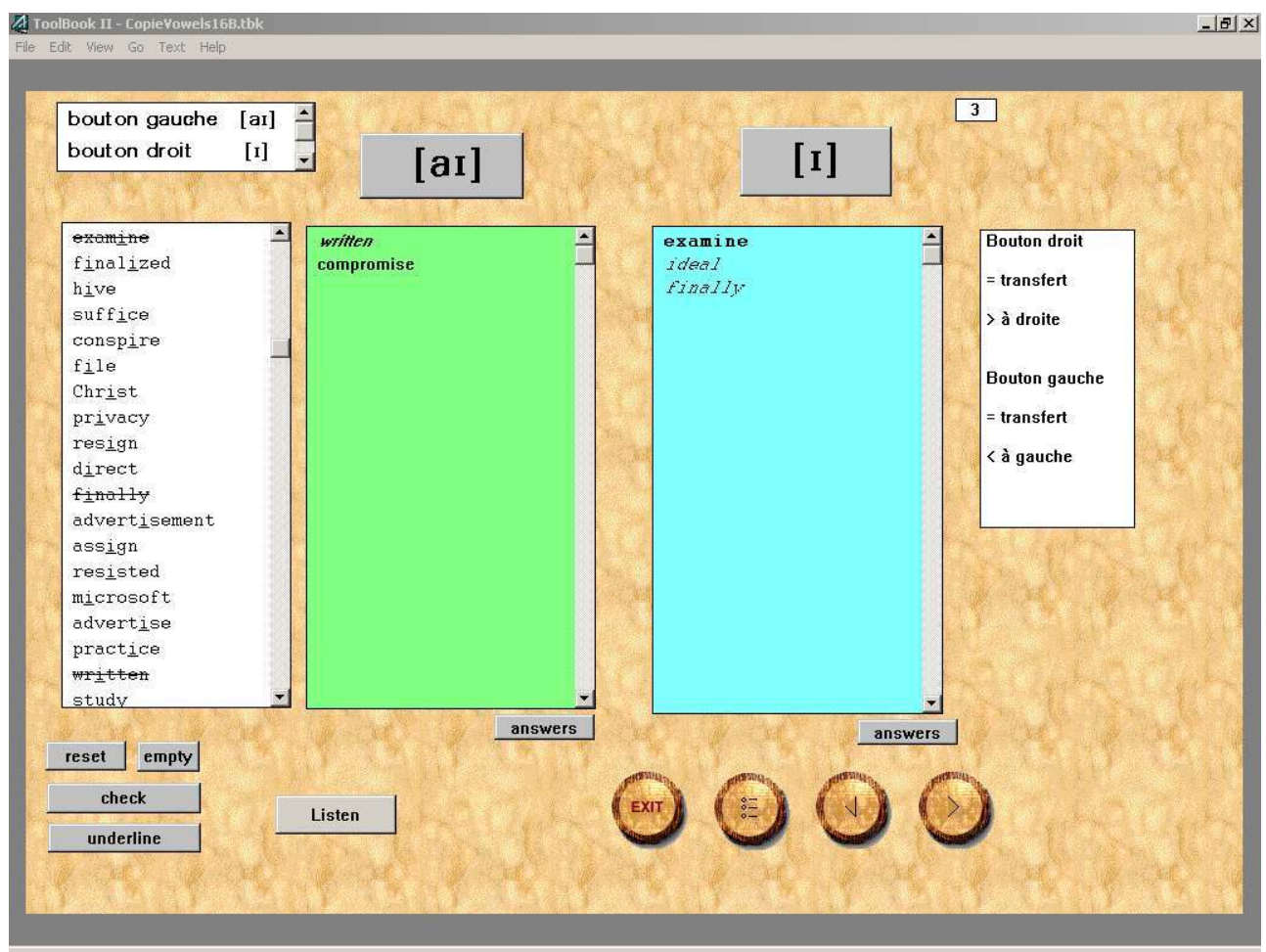

11 Les mots 'written' 'ideal' et 'finally' s'affichent en italiques, pour signaler qu'ils sont mal classés. Les mots 'compromise' et 'examine' s'affichent en gras pour indiquer des réponses correctes.

\subsection{Choix multiple pour un même graphisme}

Le but de la deuxième activité est de faire prendre conscience à l'étudiant, par exploration, des diverses prononciations d'une même graphie, ici les lettres /ea/. Un programme permet de sélectionner, dans l'un des textes juridiques stockés sur le serveur, tous les mots présentant cette graphie. La liste s'affiche dans la colonne de gauche. L'étudiant doit d'abord cliquer sur le mot dans la liste, puis sur le petit carré près du symbole phonétique. Si le choix est juste, le mot vient se positionner dans la colonne ciblée, s'inscrit en caractères gras, et est rayé de la première colonne. Si le choix est mauvais, le mot s'affiche en italiques (Fig.2). Le son est disponible en cliquant sur chaque mot avec le bouton droit de la souris. 
Fig. 2. Coping with English vowel sounds, le cas /ea/.

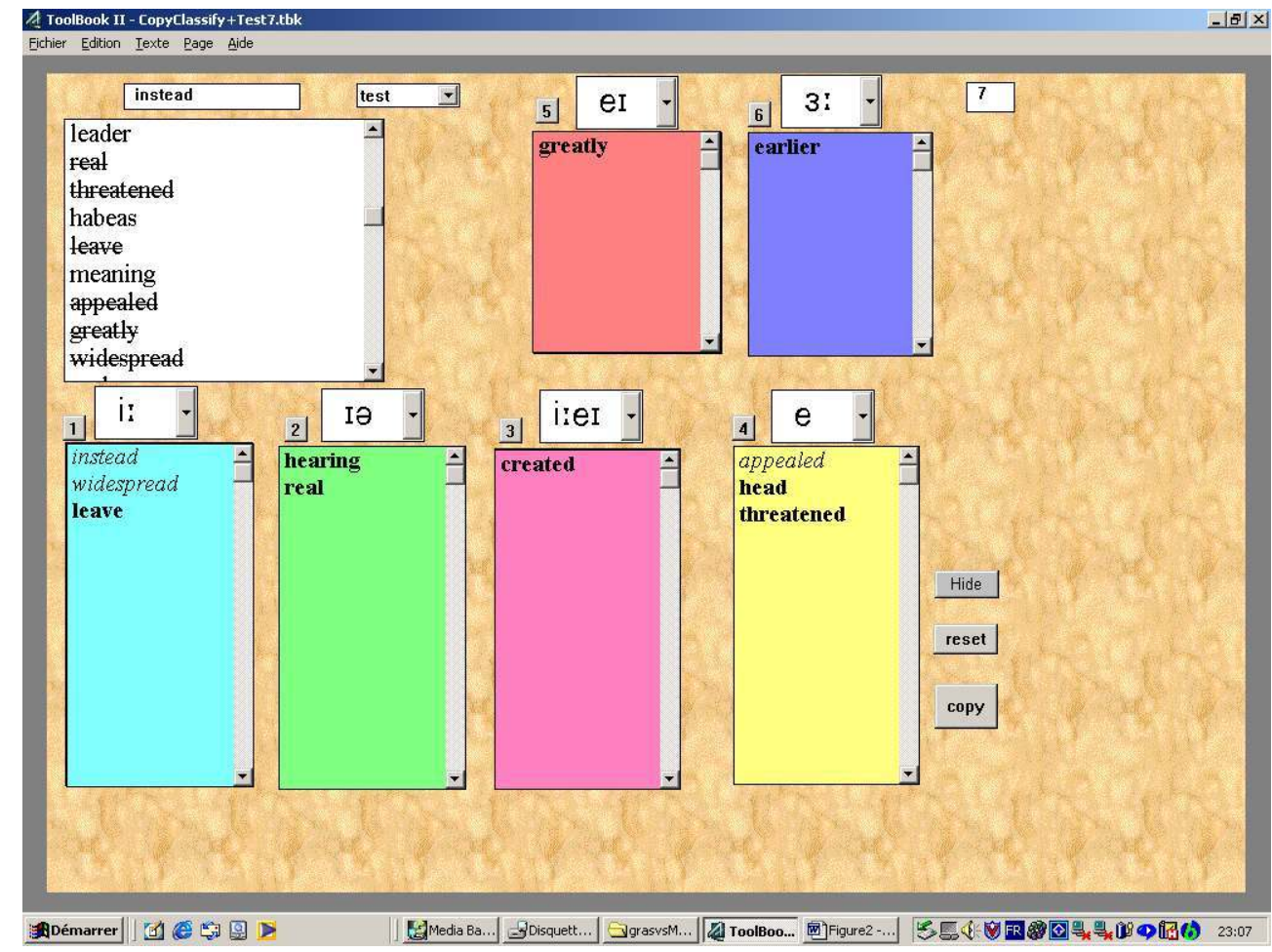

Les mots en gras sont bien classés. Les mots en italiques ('instead', 'widespread' et ' appealed') sont mal classés. Le son du mot est entendu après chaque bonne réponse. Pour corriger un mot mal placé il suffit de cliquer avec le bouton droit ou gauche pour le faire déplacer vers la colonne de droite ou vers celle de gauche.

\subsection{Création}

14 Ce programme permet à l'enseignant même néophyte d'élaborer lui-même ses exercices sans savoir programmer. Il peut par exemple, comme on le voit dans la figure 3, modifier instantanément les en-têtes des colonnes grâce à un menu déroulant qui donne accès à l'ensemble des caractères phonétiques associés aux voyelles. Pour l'utiliser et effectuer ce changement, il n'est pas nécessaire que l'enseignant maîtrise les correspondances complexes entre clavier et affichage des caractères de l'alphabet phonétique international (IPA). 


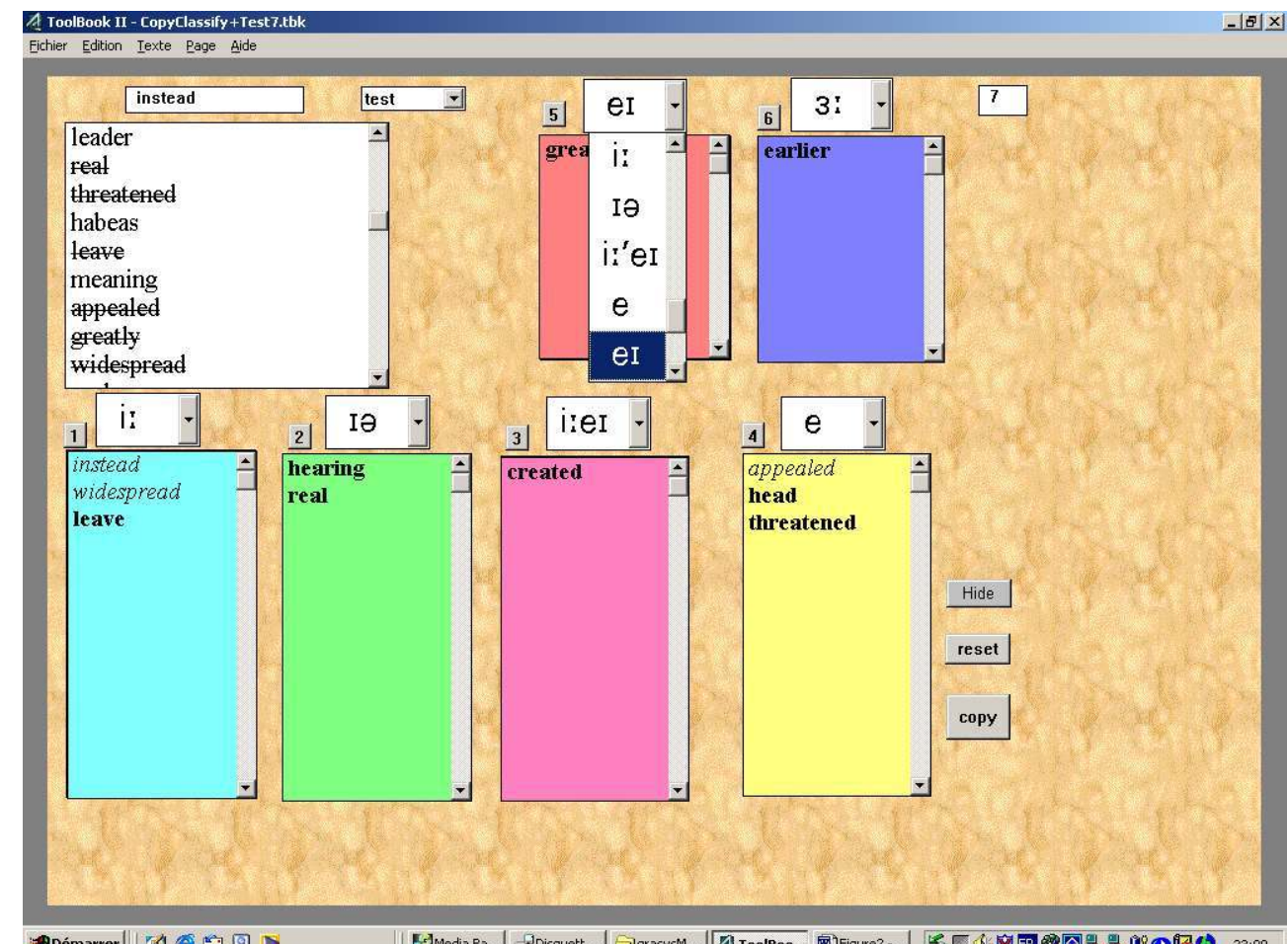

Modification de l'en-tête des colonnes par l'enseignant. Dans la colonne 5 l'enseignant est en train de choisir les caractères phonétiques qui conviennent à partir d'un menu déroulant. La sélection est simple et n'exige pas une saisie fastidieuse à l'aide du clavier.

Cet outil a été conçu comme un template, sorte de coquille vide où l'enseignant peut facilement remplacer les mots de notre liste par ceux d'une autre liste, selon la spécificité de son domaine d'enseignement. On peut aussi, sur le même principe, générer un exercice pour traiter de la terminaison des verbes réguliers au prétérit qui vont se placer, selon leur terminaison, sous les symboles [t], [d] ou [Id].

Pour générer des exercices l'enseignant passe par une page de création. Il clique sur 'first', 'second' ou 'third' pour indiquer la colonne où doivent figurer les réponses. La numérisation du son s'effectue au coup par coup et demande environ deux heures de travail pour une centaine de mots.

\section{Accent tonique}

Bien accentuer les mots en parlant est essentiel, puisque c'est autour des accents que s'organisent les schémas intonatifs de la phrase. Nous avons emprunté à Brazil (1994) son système de transcription simple et efficace, le marquage des syllabes accentuées par des majuscules. Ces annotations graphiques originales, combinées au son, vont aider l'apprenant à mieux mémoriser ce qu'il entend. Les avancées technologiques récentes permettent l'amalgame image/son sur un PC ordinaire qui devient ainsi une véritable audiothèque. Les outils de référence, tel le dictionnaire sonore incorporé dans l'encyclopédie Encarta (Microsoft) ou le programme Babylon (Babylon.com), offrent à présent un accès immédiat au son, que ce soit une voix humaine ou une voix de synthèse. 
Pour convaincre les étudiants de la nécessité d'un travail sur l'accent tonique, nous utilisons une séquence vidéo (CNN mars 2001) qui montre comment Bernard Kouchner, malgré une pratique intensive et quotidienne de la langue (au Kosovo pendant dix-huit mois), produit certaines erreurs qui entravent gravement la communication. Dans l'extrait du script présenté ci-dessous, l'accentuation de B. Kouchner est indiquée audessous des mots, et la prononciation correcte au-dessus, les majuscules signalant dans les deux cas les syllabes accentuées. À l'écran, on utilise en outre un code de couleur : rouge pour les erreurs, bleu pour les bonnes réponses.

CNN Journalist: After your experience in Kosovo, do you think that it could be an independent state, and do you personally support that?

NEcessary

B. Kouchner: Well, I think this is not necessary, it's up to the future and we'll neCESSary

see the ...in twenty or thirty years. But I'm completely convinced and resoLUtion

sure that the way, that the famous resolution 12-44, I was in charge of reSOLution implemenTAtion

the implementation of, was very clear. 'Substantial Autonomy and... impLEmentation

Un public d'enseignants anglophones dont l'opinion fut sollicitée (Association of University Language Centres, Belfast 2001) confirma notre intuition. Le seuil de tolérance aux fautes d'accent tonique est bas. Un à cinq dixièmes de seconde sont nécessaires pour comprendre le sens du mot 'resolution' si l'accent est déplacé (prononcé 'reSOLution' au lieu de 'resoLUtion'). Ce délai n'entraverait pas la compréhension, si l'erreur était isolée. Mais l'effet cumulé des fautes d'accent tonique est dissuasif. Même des enseignants de langue chevronnés « décrochaient » après quelques minutes.

Pour l'exercice de remédiation de l'accentuation, l'écran est divisé en quatre colonnes (Fig. 4). Une liste de mots du corpus figure, en minuscules, dans la colonne de gauche. L'étudiant clique sur la syllabe qu'il pense être accentuée. Si le choix est juste, le mot se déplace vers la droite dans l'une des trois autres colonnes. La syllabe accentuée apparaitt alors en lettres majuscules, tandis qu'on entend le mot prononcé automatiquement. Si le choix est mauvais, le mot reste en minuscules. 


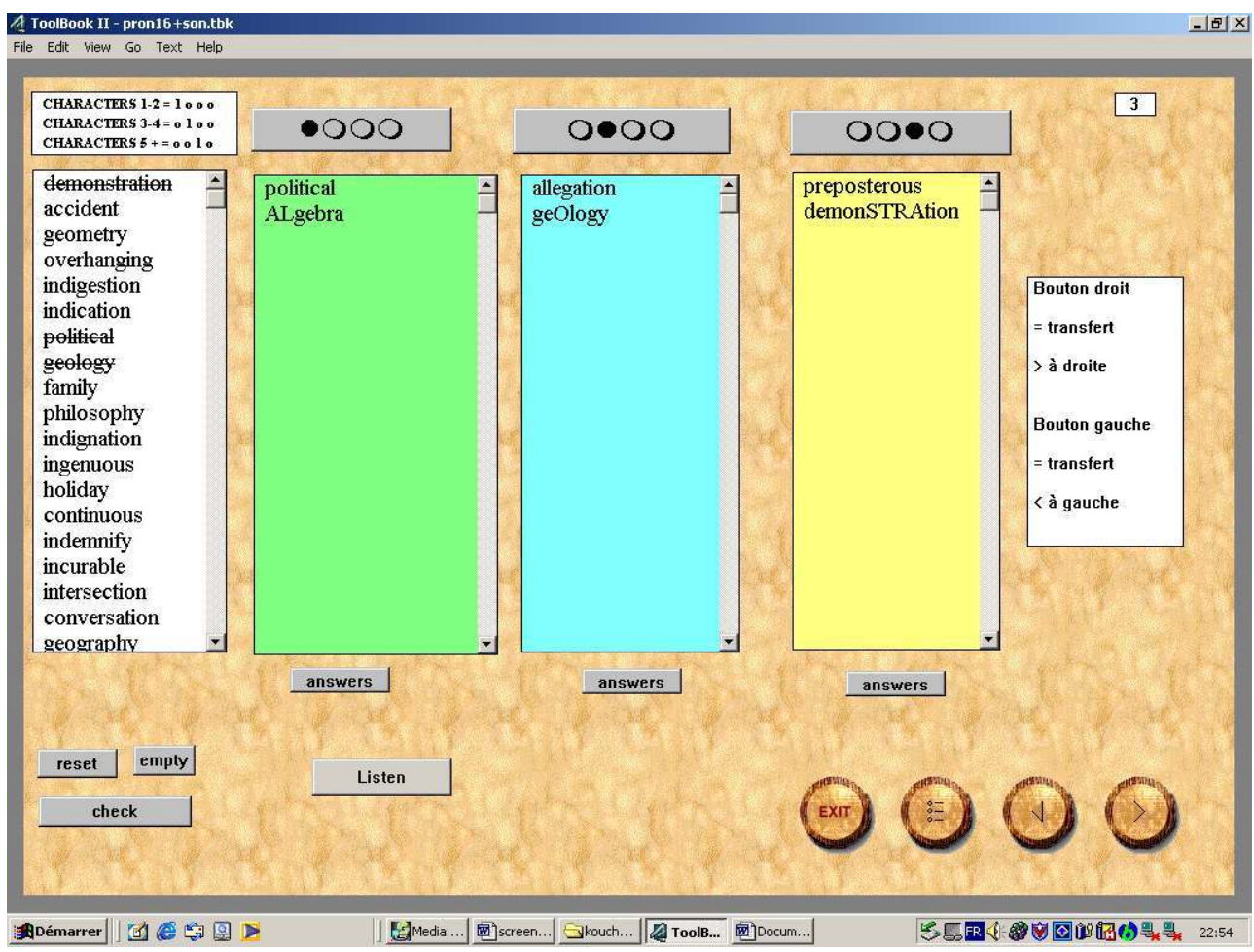

Un étudiant a déjà classé six mots dont trois sont bien placés comme les majuscules l'indiquent: 'ALgebra', 'geology', 'demonSTRAtion'. Les réponses qui s'affichent en minuscules sont erronées.

Il est également possible d'entendre le son avant de cliquer sur la syllabe, ou encore d'entendre tous les mots prononcés, avant de faire l'exercice. L'étudiant peut modifier son choix : en cliquant avec le bouton droit sur le mot pour le faire changer de colonne, le mot 'political' se place dans la colonne suivante, la syllabe 'LI' apparaît en majuscules, tandis qu'on l'entend prononcé.

Cette technique de classification présente un gain de temps par rapport au traditionnel 'glissé-déposé'. L'étudiant agit directement sur la syllabe et peut mieux se concentrer sur la place de l'accent tonique. L'inconvénient est une certaine inexactitude, dans le système de repérage, problème auquel nous cherchons actuellement une solution. On pourrait effectuer une indexation à partir d'un dictionnaire de prononciation dans une base de données pour permettre une vérification un peu moins contestable.

Stimulés par cette activité, les étudiants demandent les règles régissant l'accentuation des mots. Pour répondre à cette demande, un tableau récapitulatif simplifié figure en annexe du programme.

\section{Perspectives}

Les anachronismes des usages typographiques, dont certains sont restés inchangés depuis 1928, date où les normes de l'imprimerie moderne ont été établies, ont trouvé des critiques bien méritées dans Richaudeau (1999). Tandis que, grâce à l'ordinateur, nous expérimentons, pour souligner les traits pertinents, en jouant avec la taille des 
caractères, la couleur et les espacements, nous sommes confrontés à un grand conformisme dans la typographie. Au troisième millénaire, cependant, il ne suffit plus que la respectable profession de l'édition accepte de mettre de l'ordre dans son « fatras pseudo typographique ». L'utilisation de la couleur à l'écran et sur papier pour faciliter et améliorer la mémorisation constitue un défi pour l'éducation et le monde de l'édition. Faut-il changer les couleurs des caractères ou la couleur du fond du champ de texte, comme nous l'avons fait dans la Fig. 3 pour distinguer les six façons de prononcer les voyelles /ea/? Faut-il faire l'hypothèse d'une lecture à l'écran plus lente, mais d'une mémorisation plus efficace, suite à l'intégration de caractères en couleur, ou verra-t-on plutôt une lecture dite d'écrémage, survolant le texte pour ne prendre connaissance que des parties qui concernent la prononciation? Les protocoles de recherche sur la couleur mis en place aujourd'hui demanderont de longues années de validation, mais leur importance ne devrait pas être sous-estimée.

Dans ce genre de travail, dont la programmation est invariablement longue et laborieuse, se pose toujours la question de la pertinence de l'usage des technologies. Bien sûr l'étudiant, après avoir reçu une marche à suivre, progresse à sa propre vitesse. Mais nous devrons poursuivre nos investigations pour vérifier dans quelle mesure le multimédia présente un avantage pour l'apprentissage de la phonétique par rapport aux méthodes traditionnelles. Nous pensons d'ores et déjà qu'il y a un avantage certain à visualiser à l'écran l'accent tonique et les schémas intonatifs.

Nous insisterons, pour terminer, sur le concept d'environnement. Là où il fallait jadis un support papier, une cassette audio et un magnétophone, tout est regroupé et, si l'on y ajoute l'accès aux dictionnaires sonores que nous avons évoqués plus haut, la remédiation est incontestablement plus attrayante. Il faut par ailleurs garder à l'esprit la notion d'intégration, cette activité ne constituant qu'un des volets de l'apprentissage de l'expression orale

\section{BIBLIOGRAPHIE}

Ballier, N. 2001. «Algorithmique de l'intonation », Communication à l'atelier Nouvelles technologies, Congrès SAES, Montpellier.

Bertin, J.-Cl. 1998. « Conception de la leçon multimédia : liberté ou guidage ». ASp 19-22, 313-332.

Brazil, D. 1994. Pronunciation for Advanced Learners of English. Cambridge : Cambridge University Press.

Brazil, D. 1997. The Communicative Value of Intonation in English. Cambridge : Cambridge University Press.

Brossard, J. 1995. Enseigner la prononciation anglaise. Paris : Bordas.

Burgess, J. \& S. Spencer. 2000. «Phonology and Pronunciation in integrated language teaching and teacher education ». System 28/2,191-215.

Cazade, A. 1999. « De l'usage des courbes sonores en apprentissage des langues ». Alsic 2/2, 3-32. 
Celce-Murcia, M., D.M Brinton \& J.M. Goodwin. 1996. Teaching Pronunciation. A reference for teachers of English to speakers of other languages. Cambridge : Cambridge University Press.

Chum, D. M. 1989. « Teaching tone and intonation with microcomputers ». CALICO Journal, 21-46.

Culioli, A. 1992. La théorie d'Antoine Culioli. Paris : Ophrys.

Germain, A. \& Ph. Martin. 1999. «WinPitch Language teaching and learning: écouter, voir et manipuler la production orale pour l'apprentissage en langue seconde ». Jila, Nice 24-25 juin. Publication de la Faculté des lettres de l'Université de Nice-Sophia-Antipolis, 110-114.

Germain, A. \& Ph. Martin. 2000. « Présentation d'un logiciel de visualisation pour l'apprentissage de l'oral en langue seconde ». Alsic 3/1, 71-86.

Gilbert, J. 1993. Clear Speech: Pronunciation and listening comprehension in North American English. Cambridge : Cambridge University Press.

Ginésy, M. 2000. Phonétique et phonologie de l'anglais. Paris : Ellipses.

Krashen, S. 1983. " The din in the head, and the language acquisition device ». In Oller, J. W. Jr \& P. A. Richard Amato (dir.), Methods that work. Rowley, MA : Newbury House.

Lilly, R. \& M. Viel. 1993. Initiation à la phonétique de l'anglais. Paris : Hachette.

Murphy, R. 1996. English Computerized Learning Inc., Pronunciation Power. Edmonton, Canada : Blackstone Multimedia Corporation.

Papert, S. 1994. L'enfant et la machine à connaître. Paris : Dunod.

Péchou, A. \& A. Stenton. 1999a. « Contribution de la vidéo numérique pour améliorer la compréhension et l'expression orale. Compte-rendu d'une expérimentation à partir de séquences $\mathrm{du}$ film The Firm ». Les Après-midi de LAIRDIL 10, 9-15.

Péchou, A. \& A. Stenton 1999b. « Hypermedia, concordances and filters for language learning in the social sciences ", Actes des Journées internationales de linguistique appliquée, 24-25 juin, Laboratoire d'Ingénierie Linguistique et de Linguistique Appliquée (LILLA), Université de Nice Sophia Antipolis.

Péchou, A. \& A. Stenton. 2000. «Utilisation des images fixes pour les présentations assistées par ordinateur ». ASp 27-30, 393-400.

Péchou, A. 2001. « Approches de l'anglais du droit et de l'économie à l'université de Toulouse 1 ». In Mémet M. et M. Petit (dir.), L'anglais de spécialité en France, Mélanges en l'honneur de Michel Perrin. Bordeaux : Éditions du GERAS, 159-172.

Peperkamp, S. 2001. « Typologie des langues accentuelles : perspectives, développementales et données comparatives ", Troisièmes journées internationales du GDR 1954 'Phonologie', Nantes 30 mai-1 ${ }^{\text {er }}$ juin 2001.

Richaudeau, F. 1999. Des neurones des mots et des pixels. Rellianne (04110) : Atelier Perrousseaux.

Roach, P. 1991. English Phonetics and Phonology : A practical course. Cambridge : Cambridge University Press.

Rossi, M. 1999. L'intonation, le système du français : description et modélisation. Paris : Ophrys.

Stenton, A.J. 2000. « Le projet TUSSELLE, l'utilisation des filtres linguistiques pour l'apprentissage d'anglais dans les sciences sociales », Actes du Groupe de linguistique appliquée de Télécom, ENST Brest, 11-13 juillet 2000. 


\section{NOTES}

1. English Computerized Learning Inc., Alberta, Canada.

2. L'Université Toulouse 1 a introduit une cyberlicence en Droit, enseignée à distance, entre Toulouse et Albi, qui a obtenu le prix des Trophées de la Nouvelle économie, le 29 juin 2000. Cette formation, qui permet de délivrer une licence en bonne et due forme, s'appuie en effet, dans le cadre du télé-enseignement, sur la mobilisation de l'ensemble des outils multimédias : PC reliés via Internet à la faculté de Toulouse équipée en système serveur, systèmes audio et visioconférences, didacticiels sur cédéroms, etc.

\section{RÉSUMÉS}

La mémoire visuelle est la plus efficace des mémoires sensorielles, mais peut-elle aider les étudiants à améliorer leur prononciation de l'anglais? Nous avons cherché à répondre à la question en utilisant un ensemble d'innovations typographiques à l'écran couplées à des enregistrements sonores numériques de qualité. Convaincre le public de l'étendue du problème à travers des vidéos de francophones parlant anglais, en affichant les fautes, n'était que la première étape. Le système auteur développé pour générer des exercices de classification est le fruit de plusieurs années de recherches sur la langue des sciences sociales, rassemblant dans un corpus deux types d'erreurs : discrimination voyelles/diphtongues, et place de l'accent tonique dans le mot. La validation précoce du prototype conçu pour l'auto-apprentissage et l'apprentissage à distance suggère des possibilités intéressantes pour aider à la remédiation et au diagnostic.

Visual memory is the most efficient sensorial memory, but can it be used to help students improve their pronunciation? This paper outlines an innovative approach to the question involving computer screen typographical experiments combined with high quality digital sound recordings. Convincing the learners of the seriousness of the problem through use of a francophone videobank of displaced main stress errors was just a starting point. The development of an authoring system for generating pronunciation classification exercises is the fruit of several years of research in the language of social sciences and a corpus of errors: vowel/ diphthong discrimination and the place of the stress. Early prototype testing which includes selfstudy and distance learning applications indicates progress in helping remediation and diagnosis.

\section{INDEX}

Mots-clés : accentuation de mots, apprentissage des langues, multimédia, phonétique, typographie

Keywords : language learning, pronunciation, word stress, typography 


\section{AUTEURS}

\section{ANNE PÉCHOU}

Anne Péchou est Professeur à l'université Toulouse 1. Elle est spécialiste de la didactique de l'anglais oral et du travail de groupe. Sa thèse de doctorat sur les moyens de surmonter les difficultés à s'exprimer en langue étrangère dans le contexte de la classe comporte des approches neurolinguistique, psycholinguistique et pragmatique. Elle s'intéresse aux styles d'apprentissage, et travaille actuellement sur l'utilisation des nouvelles technologies pour la compréhension de l'oral en laboratoire multimédia. Elle a créé plusieurs modules multimédia pour la langue de l'économie et du droit avec le logiciel Smart Alex d'A. Stenton. Elle a encadré des DEA sur la didactique, l'oral et l'enseignement à distance. Pechou@univ-tlse1.fr

\section{ANTHONY STENTON}

Anthony Stenton est maître de conférences à l'université Toulouse 1, et titulaire d'un M.A. de Cambridge University. Il est l'auteur de nombreux logiciels pour l'apprentissage des langues et spécialiste des études linguistiques de corpus et de l'hypermédia. Sa thèse de doctorat, décernée par l'université de Bourgogne en 1992, concerne l'apport des nouvelles technologies de l'information et de la communication à l'apprentissage de l'anglais. Il est responsable du Laboratoire multimédia de l'université Toulouse 1 et responsable du Focus Group 'NTIC' pour CERCLES (Confédération Européenne des Centres de Langues de l'Enseignement Supérieur). Il a développé plusieurs logiciels : Smart Alex, système auteur hypermédia, Legal English Workshop et Coping with Stress en collaboration avec A. Péchou. Stenton@univ-tlse1.fr 